\title{
A Review of Nanoliposomal Delivery System for Stabilization of Bioactive Omega-3 Fatty Acids
}

\author{
Zahra Hadian
}

National Nutrition and Food Technology Research Institute (NNFTRI), Shahid Beheshti University of Medical Sciences (SBUMS), Tehran, Iran

Type of article: Review

\begin{abstract}
Currently, bioactive compounds are required in the design and production of functional foods, with the aim of improving the health status of consumers all around the world. Various epidemiological and clinical studies have demonstrated the salutary role of eicosapentaenoic acid (EPA, 22:6 n-3) and docosahexaenoic acid (DHA, 22:5 n3 ) in preventing diseases and reducing mortality from cardiovascular diseases. The unsaturated nature of bioactive lipids leads to susceptibility to oxidation under environmental conditions. Oxidative deterioration of omega-3 fatty acids can cause the reduction in their nutritional quality and sensory properties. Encapsulation of these fatty acids could create a barrier against reaction with harmful environmental factors. Currently, fortification of foods containing bioactive omega-3 fatty acids has found great application in the food industries of different countries. Previous studies have suggested that nano-encapsulation has significant effects on the stability of physical and chemical properties of bioactive compounds. Considering the functional role of omega-3 fatty acids, this study has provided a literature review on applications of nanoliposomal delivery systems for encapsulation of these bioactive compounds.
\end{abstract}

Keywords: Omega-3 Fatty Acids; Stabilization; Nanoliposome; Bioactive

\section{Introduction}

Polyunsaturated fatty acids (PUFA) with multiple double bonds exist in the form of $\alpha$-linolenic acid, eicosapentaenoic acid, and docosahexaenoic acid. In recent decades, clinical studies have verified that increasing the content of fatty acids in diet could be effective on the lipoprotein-lipid index. Since 1970, various studies have been conducted to assess the effects of omega-3 fatty acids derivatives on preventing diseases, especially cardiovascular diseases, so that manufacturers have been encouraged to formulate dietary supplements from these compounds using different methods, including encapsulation. Various studies conducted in different communities suggest that daily intake of $1 \mathrm{~g}$ fish oil (containing about $840 \mathrm{mg}$ DHA+EPA) could significantly decrease rates of sudden death in patients with cardiovascular diseases. Therefore, using this supplement is recommended for use alongside anticoagulant drugs in such cases (1-3). The Food and Drug Administration (FDA) of America does not recommend acquiring these fatty acids through only fish consumption, because of seafood containing compounds such as polychlorinated biphenyls (PCBs), mercury, and other contaminants. In addition, the FDA has approved the use of Lovasa (Omacor), which contains EPA and DHA, as a medicinal supplement of omega-3 fatty acids because of its efficacy in preventing cardiovascular diseases in patients with high triglyceride (4-6). The unsaturated nature of omega-3 fatty acids results in their susceptibility to oxidation under environmental conditions. Hydroperoxides are products generated from the primary oxidation of EPA and DHA, followed by degradation into secondary oxidation compounds, including volatile aldehyde compounds. By encapsulation of these fatty acids, a barrier can be created to prevent reaction of these compounds with oxidative factors. When designing capsules, some factors should be considered when recognizing the target cell and stability against $\mathrm{pH}$ changes of digestive system through adding chemical compounds or other protective groups. As delivery systems, current liposomes include a wide range of

\section{Corresponding author:}

National Nutrition \& Food Technology Research Institute (NNFTRI), Shahid Beheshti University of Medical Sciences (SBUMS), 7 West Arghavan, Farahzadi Blvd., Shahrak Qods, Tehran, Iran.

Tel: +98.2122376473, Fax: +98.2122360660, e-mail: z_hadian@sbmu.ac.ir

Received: October 18, 2015, Accepted: December 20, 2015, Published: January 2016

iThenticate screening: December 20, 2015, English editing: January 02, 2016, Quality control: January 05, 2016

(C) 2015 The Authors. This is an open access article under the terms of the Creative Commons Attribution-NonCommercialNoDerivs License, which permits use and distribution in any medium, provided the original work is properly cited, the use is non-commercial and no modifications or adaptations are made. 
bioactive compounds with different applications in food, pharmaceutical, and agricultural industries (7-9). Liposomes are made of natural lipids; therefore they are non-toxic and do not stimulate the immune system, in addition to being biodegradable. Other benefits of liposomes include solubility, capacity for controlled, directed and purposeful release of hydrophobic and hydrophilic compounds, and ease of permeation and transmission through membranes. Liposomes applications include acting as suitable carriers of different bioactive molecules in all the nanomedicine platforms and liposomes have generated a great deal of recent interest. There are several formulations approved by the Food and Drug Administration (FDA) for disease treatment (10-13). Nanoliposomes can be defined as lipid bilayers assemblies encompassing the aqueous compartment within the nano-sized range. Nanoliposomes have been tried for various applications as drug or gene delivery for treatment of diseases. Various methods typically used to fabricate liposomes include the thin layer film hydration method, the ethanol injection method, and the detergent removal method, as well as the heating method, the reverse phase evaporation method, and homogenization $(13,14)$. Recently conducted studies suggest that the methods used to provide liposomes have had an important effect on the stabilization of the physical and chemical properties of bioactive compounds. In addition, by reducing the particle size of this colloidal system, the specific surface area could lead to more favorable compliance and bioavailability properties of bioactive compounds $(3,15,16)$. Selecting the method of liposome preparation depends on the following parameters: the physicochemical characteristics of liposomal lipids and those of entrapped material; the kind of dispersion medium for lipid vesicles; the concentration and potential toxicity of entrapped material; vesicle size, polydispersity, and shelf - life intended for each application; batch - to - batch reproducibility; safe and efficient liposome production on a large scale $(13,17,18)$.

\section{Effects of Omega-3 Fatty Acids on Health}

The main omega- 3 fatty acids, in terms of nutrition, are $\alpha$-linolenic acid, eicosapentaenoic acid, and docosahexaenoic acid. Technically, EPA is not essential fatty acid, since the body can convert essential $\alpha$-linolenic acid into EPA. However, the physiologic conditions of the human body cannot create DHA and EPA sufficiently to supply them as required for healthly living and these compounds should be provided through dietary sources. Omega-3 fatty acids are obtained from seafood, oils (algae oil, fish oil and flax seed oil), or dietary supplements. EPA is found in fish and fish oil. DHA is of special importance for the body and is found mainly in fish and commercial algae oil. ALA is mainly found in seeds, herbal oils, and green leafy vegetables. In the body, ALA is first converted to EPA and then to DHA (19). Fish oil is often accessible in the form of soft-gel capsule with different commercial names. Vitamins, antioxidants, and different flavors are added to these products. Lovaza is a type of fish oil supplement with a high concentration of omega-3 (465mg EPA and 375mg DHA), which was approved by the FDA in 2005 as a supplementary treatment for patients with hypertriglycemia $(1,20)$. DHA is the main ingredient in the phospholipid membranes of the brain and retina cells. In addition, DHA decreases serum triglyceride by inhibiting lipogenesis and simulates oxidation and lipid metabolism. EPA and DHA, in omega-3, are significantly effective for reducing plasma TG. By simulating peroxisome proliferators-activated receptor, DHA and EPA increase lipid metabolism and decrease serum level of triglyceride. In addition, EPA is capable of reducing total cholesterol $(4,8)$. The protective effects of unsaturated omega-3 fatty acids, such as EPA, in coronary heart diseases are applied through different methods, some of which involve over activation of the sympathetic nervous system, enhancement of vasodilation through reduction of endothelial inflammatory response by inhibition of monocyte adhesion to the wall of arteries, and weakening the production of inflammatory mediators and antithrombotic action. Overall, the beneficial effects of omega-3 fatty acids on coronary arteries significantly reduces sudden death in patients with cardiovascular diseases and specialists often recommend using supplements along with antiplatelet drugs, beta blockers, and angiotensin converting enzyme inhibitors (20). In 2000, the FDA approved the use of fish oil supplements for reducing the risk of cardiovascular diseases and recommended patients with coronary artery disease to take $1 \mathrm{~g}$ of supplements daily (21).

\section{Stabilization of Omega-3 Fatty Acids, Characterization Method and Control of Autoxidation}

The major factors in stabilization of lipids include the type of ingredients of fatty acids, temperature, oxygen, humidity, heavy metals, light, and packaging. Various lipid oxidative reactions also can occur, including nonenzymatic autoxidation, light oxidation and enzymatic oxidation. In this regard, autoxidation is the main process and non-enzymatic autoxidation of EPA and DHA occurs in ambient temperature, due to the high unsaturated nature of the compounds, which causes further changes during processing. These fatty acids oxidize rapidly with exposure to light, peroxidants, or high temperature, which causes primary oxidative products to be formed. Sensory changes resulting from degradation of hydroperoxides into secondary products, including aldehydes, ketones, acids, and alcohols, are proportional to the carbon chain length and degree of unsaturation for the fatty acids. Some of these compounds have olfactory thresholds. Therefore, they affect sensory quality at very low concentrations and result in 
unpleasant taste and odor (22). Lipid oxidation is considered the main parameter affecting the retention of different fish oils and products containing it. Autoxidation of lipids results in formation of fatty acid based hydroperoxides and their degradation into secondary oxidation products. Many of these compounds are the cause of unpleasant tastes and further degradation. Some methods for preventing oxidation of unsaturated lipids include reduction of oxygen through proper packaging, storage at low temperatures, and addition of antioxidants (23). The use of nanodelivery systems, such as liposome, can stabilize and protect bioactive compounds including omega-3 fatty acids against changes in environmental conditions and chemical changes, along with improving their accessibility. Researchers believe that omega-3 fatty acids could be the best option of functional foods, if the oxidative deterioration difficulties are overcome (16).

\section{Encapsulation Techniques of Omega-3 Fatty Acids}

Encapsulation and controlled release techniques have developed very quickly in the last three decades throughout the medical and pharmaceutical industries. In general, microencapsulation technologies are divided into three groups, namely chemical methods of molecular inclusion and interfacial polymerization, physical methods including spray drying, freeze drying, air suspension coating and extrusion, as well as physicochemical methods such as coacervation, inclusion complex, and liposome entrapment (16). In the food sector, the main objectives of encapsulation technology are protecting active compounds, covering undesirable properties and controlled release of materials. New microencapsulation technologies are continuously being developed in functional food markets and are the main innovations in this field (24). Currently, products from the nanotechnology market comprise over one billion dollars in the food industry and this will develop to over 20 billion dollars in next decade. Two methods for accessing nanoparticle systems include mechanical or top-down methods, which work by reducing particle size and chemical processes, or bottom-up methods, where nanoparticles are created from smaller molecules, such as lipids or proteins (25). Encapsulation methods are capable of affecting the color, shape, weight, volume, solubility, sensitivity to pressure, and sensitivity to heat and light of compounds. Nanotechnology is used for designing, characterization, production and application of structures, devices and nanosized devices and systems $(<100 \mathrm{~nm})$. Nanoencapsulation is a new method to protect bioactive compounds (from harmful environmental conditions, oxidation, pH change and enzymatic degradation) such as omega-3 fatty acids and keep them available as functional food compounds. Compared to microencapsulation, nano-sized delivery systems provide more surface, increased solubility, improved bio-availability, and improvement of controlled release, as well as active targeting (26-29). Using capsules or encapsulation of fatty acids with multiple double bonds, a barrier can be created to prevent the oxidation of these compounds. Efficiency of encapsulation and stability when maintaining microcapsules of omega3 oil is a point of interest in developing encapsulation systems. Accessing these features depends on selection of formulation and the processing conditions used to produce microcapsules (28).

\subsection{Liposome as Delivery System of Functional Compounds}

Biologically, liposomes can deliver bioactive compounds, since they are made from natural lipids. Therefore, while being biodegradable, they are non-toxic and do not stimulate the immune system. Their stability could be enhanced as well, since bioactive compounds are trapped inside liposome (30). Currently, liposomes, as delivering systems, comprise a wide range of bioactive compounds and have various applications in the food industry, the pharmaceutical industry, and agriculture. The oldest studies using liposomes in the food industry date back to 1980 and investigate liposomes as carriers of proteins, pigments, vitamins, antioxidants, and flavoring agents. Other applications of liposomes in the food industry involve encapsulation of protease enzyme that prevents premature breakdown of casein and reduction of enzyme loss. Encapsulation of lactase is another application of liposome that releases lactase in the stomach and, therefore, removes the sweet taste of hydrolyzed milk $(9,17,27,31)$. Because of its many advantages, using liposome as carrier for bioactive compounds was approved by the FDA, in addition to approval of loaded medical compounds inside liposomes, relative to using them as free from. Many products in the form of liposome are available and have been approved by the FDA for cancer treatment (32). Liposomes are created from one or more phospholipids, as one or more bilayer membranes, are capable of encapsulating compounds with low molecular weight, proteins, and genes. Various methods are used to produce liposomes. Some common methods include thin layer film hydration, detergent depletion, ethanol/ether injection, reverse phase evaporation, and emulsification methods $(3,18)$. In all methods, the lipid phase, which is typically an organic phase, is mixed with the aqueous phase $(3,33)$. In most mentioned methods, liposomes as multilamellar large vesicles (MLVs) are formed and to obtain suitable and uniform particle size, various methods are used, including extrusion, sonication, and homogenization (3). Selecting suitable method to produce liposomes and encapsulate bioactive compounds first depends on the physicochemical properties of the material in question. Bioactive compounds are 
divided into two classes depending on their interaction with liposome membranes: 1) Water soluble compounds that are easily encapsulated inside the aqueous phase at the center of liposomes or the aqueous phase between layers; 2) Amphiphilic and lipophilic compounds have been shown to be trapped in the liposomal bilayers only if they formed a complex with the lipid layers acyl chains. It is the coefficient of fat distribution or, in other words, the coefficient of liposome distribution that causes a compound to be in the first or second group. In the case of some materials, a method could be selected depending on the $\mathrm{pKa}$, composition, and charge of the phospholipids, so that the intended compound enters liposome aqueous phase or accumulates in the lipid bilayer (34).

4.1.1. Stabilization of Liposomal Carrier Systems

Stabilization of colloid systems is typically described based on the presence or lack of particle surface potential. Surface potential is created when dispersed solid particles have an electrical charge relative to their ambient aqueous environment. Solid particles can obtain electrical charge through a variety of methods. In brief, the main mechanisms involved in creating particle charge are as follows: 1) Ionization of surface groups: Ionization is a common mechanism for surfaces with ionizing groups. Some examples are carboxylic acid, sulfuric acid, sulfonic acid esters, amino groups, and ammonium groups. In these cases, the amount of particles' charge depends on pH of the carrier environment; 2) Surface ion adsorption: In this state, adsorbed ions create surface electric charge in particles. Surface adsorbed surfactants may create charge in particles through ionization. In colloid systems such as liposomes, electrostatic repulsive forces, Van der Waals attraction, and spatial repulsive forces play a more prominent role in stabilization of the system (35). Stabilization of colloid systems is often performed based on electrostatic repulsion (DLVO theory), spatial repulsion (HVO: Hesselink-Vrij-Overbeek theory), and a combination of electrostatic and spatial repulsion.

4.1.1.1. Physical Stability

By forming a liposome, part of aqueous phase is entrapped inside a close and continuous bilayer construction and, therefore, water-soluble compounds could be encapsulated inside the liposome. In addition to entrapment of polar compounds in the aqueous phase of the vesicles, entrapment of hydrophobic and hydrophilic compounds can be solved via formation with the bilayer lipid or reaction with this lipid layer. This trend can change vesicle properties, including the permeability and stability of bilayer construction. Stability of liposome formulations during construction, maintenance, and consumption is an important factor in selecting liposomes as proper carriers of bioactive compounds. Stability of the molecular structure of liposome membrane and materials entrapped by the liposome are both important to consider. In the case of compound formulation of liposomes, 2 -year stability in $4 \mathrm{oC}$ is considered favorable. Stability of liposome membrane depends on the arrangement of hydrocarbon chains of the lipid molecules $(10,37)$. Some of the physical changes in liposomes include changes in particle size, due to aggregation or fusion during maintenance because chemical or thermal decomposition reactions or leaking of encapsulated material. Physical stability typically includes fixed particle size and ratio of loaded compound into liposome lipids and it is observed that at optimized $\mathrm{pH}$ and suitable salt concentration, the physical properties of liposome remain constant in refrigerator temperature (38).

4.1.1.2. Chemical Stability

Chemical instability in liposomes is mainly the result of hydrolysis of ester groups and oxidation of unsaturated lipid acyl chains. During hydrolysis, the ester bond is broken and hydrophobic chains are separated from the lipid. In addition, $\mathrm{pH}$, temperature, and buffer ingredients are effective on hydrolysis kinetics. Studies reveal that changes in acidity or alkalinity accelerate hydrolysis reactions. Oxidation occurs because of the presence of unsaturated phospholipids in the liposome structure. Using natural or synthetic antioxidants, including $\alpha$-tocopherol, could effectively solve this problem (38). During oxidation reactions, the structural and physical behavior of bilayers can altered significantly. The peroxidation of phospholipids occurs in the same way as peroxidation of triacylglycerol. This fact means that change in configuration of the phospholipid acyl chain brings about important changes in the structure of the liposomal membrane. Physical properties and arrangement of lipid molecules are factors that affect oxidation of liposome bilayer membrane. Lipid peroxidation occurs through a series of sequential reactions. It is obvious that the size and z-potential of liposomes are influenced by the peroxidation reaction (39).

4.1.1.3. Biologic Stability

Changes occur in liposome structure that contact biologic systems in an undesirable manner. The main biologic instability of liposomes is their high tendency to aggregate, as well as drug release in plasma. This problem becomes more complicated in vivo because of the presence of surfaces with negative charge, colloid systems, and main ingredients of serum (38). When liposomes enter the body, they become diluted by biologic fluids, including blood, lymph, and extracellular fluids. Plasma makes up about 55\% of the body's total blood volume and is composed of various molecules, macromolecules, and different ions. In this environment, lipoproteins and phospholipase have a greater effect on liposome stability. Liposome and protein interactions result from electrostatic attraction between different surface charges or hydrophobic forces cause weak binding of proteins to the surface of the liposome or 
penetration into membrane layers of the liposome. Stability of liposomes in plasma depends on their relative concentration, size, number of lipid layers, and coordination lipid composition with the biologic environment of body and ambient temperature (40).

\subsection{Review of Literature}

Encapsulation is a process where small solid particles, liquid droplets, or gas are trapped in encapsulation materials. Encapsulation techniques have many applications in the food industry and other industries. In the food sector, the main objectives of encapsulation techniques are protecting active compound, covering undesirable properties, and controlled release of materials. New microencapsulation methods are continually being developed in the market of functional foods and are the main driving force for innovations in this field. Overall, encapsulation techniques are divided into 3 groups: chemical, physical, and physicochemical techniques (24, 41). Transferring lipophilic bioactive compounds as liposome nanoparticles is an encapsulation system developed in numerous different industries, but used especially often in the pharmaceutical industry, and recently some applications of this method have been reported in food industry as well. The oldest studies on applications of liposome in the food industry date back to the 1980s (16). Some applications of nanotechnology in the food industry include production of preservatives, oxidation-reduction factors, pigmentation compounds, sweeteners, enzymes, antioxidants, acids, acids, alkalis, buffers, flavoring agents, and nutritional compounds. The advantage of encapsulation with liposomes relative to other methods is that in the case of excessive aqueous activity in the environment, encapsulated soluble materials are not released and are only released in a target area or specific location (7). Nanoliposome technology provides exciting opportunities for food technology in areas including encapsulation and controlled release of food compounds, along with increasing bioavailability, stability, and increased shelf-life of ingredients. Liposomes are used in the food industry to transfer flavoring and nutritional compounds as well as antimicrobial materials. Currently, the market of nanotechnology products in the food industry is about 1 billion dollars and in next decade it will develop to over 20 billion dollars. Two ways of accessing nanoparticle systems include mechanical or top-down methods, which work by decreasing particle size, and chemical or bottom-up processes, where nanoparticles are generated from smaller molecules such as lipids or proteins (25). One of the applications of liposome in the food industry is encapsulation of protease enzyme that prevents premature breakdown of casein and reduces enzyme loss. Another application is lactase release in the stomach that removes the sweet taste of hydrolyzed milk. Encapsulation of various vitamins, such as vitamin $\mathrm{D}$ to fortify milk and its products, maintaining antioxidant activity of vitamin $\mathrm{C}$, and $\alpha$-topopherol, as well as encapsulation of antimicrobial materials such as nisin, are among other applications of liposome in food industry $(9,27,31)$. With regard to the relationship between consuming omega-3 fatty acids and reduced risk of cardiovascular diseases, researchers have conducted many studies on stabilization and bioavailability of omega-3 fatty acids in liposome systems. Studies by Nara et al. (42) demonstrated that marine phosphatidylcholine is very susceptible to degradation, in terms of oxidative stability. Oxidative stability of phosphatidylcholine in salmon increases with addition of cholesterol, stearylamine, and dicetyl phosphate. In addition, through using the proper amount of $\alpha$-tocopherol and albumin, oxidation of phosphatidylcholine can be prevented. The most stabilized encapsulated form of triglycerol with high DHA percentage was prepared with a liposome formulation provided from salmon egg phosphatidylcholine. This study presents the potential of liposomal formulation for encapsulation of the compounds prone to omega-3 oxidation (42). Nacka et al. evaluated the physical and chemical stability of liposomes based on marine lipids under acidic condition. Results demonstrated that acidic environments result in an increase in unilamellar fusion of phosphatidylcholine vesicles, increase the number of large unilamellar liposomes, and that the greatest effect was observed around the isoelectric point of phosphatidylcholine. In addition, findings showed that increasing the surface charge of liposomes, as well as decreasing electrolyte concentration, could interfere with decreasing fusion and aggregation (43). In a study, Nacka et al. investigated fusion of $\alpha$-tocopherol in liposomes based on marine lipids in vivo and in vitro. The results of their study demonstrated that the best oxidative stability was related to liposomal formulation, where the $\alpha$ tocopherol content was $5 \mathrm{~mol} \%$. In addition, the production of propanal was lower relative to other formulations. Liposome instability under acidic conditions ( $\mathrm{pH}$ 1.5) was evident along with hydrolysis (44).

Moussaoui et al. investigated the physical properties of liposomes provided by marine lipids (Marionosomes). Liposome structural properties, including turbidity measurements via spectrophotometry of optical density at 400nm (evaluation of morphology stability), granulometric measurements to determine accurate size (equal to $300 \mathrm{~nm}$ to 0.2 $\mu \mathrm{m}$ after filtration), and observing spherical shape using phase-contrast microscopy, were verified. In addition, using freeze-fracture transmission electron microscopy, liposomes were evaluated in terms of the number of lamella and whether they were all oligolamellar. Storing liposome suspensions for 30 days at ambient temperatures indicated its 
stability. However, by reducing $\mathrm{pH}(\sim 4)$ and buffer, and using high concentration of calcium, liposome size decreased and liposomes aggregated (45). After providing liposomes based on natural marine lipid by Cansell et al., availability of omega-3 fatty acids in animal models showed that marine phospholipid could be an attractive compound to produce liposomes as food supplements with PUFA (46). Lyberg et al. surveyed oxidation of different forms of docosahexaenoic acid within 28 days of storage at $28-30 \mathrm{oC}$. Based on the results of their study, phospholipid protects oxidation of carbon 4, 7, 8, and 11 of DHA. In addition, DPPC significantly prevents oxidation of carbon 20 of free DHA, which is the most prone position to oxidation. This study shows the effective antioxidant ability of DPPC phospholipid. This property is related to the arrangement of DHA atoms that create a special bending and curvature in this molecule (47). Onuki et al. evaluated the effects of fatty acids on thr physical properties of model bilamellar lipid membrane. Applying stearic acid (SA), oleic acid (OA), docosahexaenoic acid (DHA), and eicosapentaenoic acid (EPA) in lipid formulations decreased Van der Waals reactions between hydrocarbon chains of phospholipid because of a twisted structure related to their double bonds (48). In another study, Namani et al. demonstrated that when the molecular ratio of neutral DHA to anionic DHA is between 1:3 and $1: 3$ ( $\mathrm{pH}$ between 8.5 and 9.2 and $10 \mathrm{~m} \mu \mathrm{DHA}$ ), it is shaped to form a vesicle. Investigating DHA vesicles using cryo transmission electron microscopy (Cryo-TEM) demonstrated that the width of a bilamellar vesicle was less than two times the length of a DHA molecule, which shows the flexibility of DHA inside bilamellar vesicles. Comparison of vesicles prepared from saturated fatty acids, including decanoic acid, with DHA vesicles demonstrated that the DHA vesicles were chemically less stable as a result of the 6 allylic double bonds. Using calcein illustrated that watersoluble molecules could be encapsulated inside DHA vesicles. Therefore, their application in medicine and foods was suggested (49). Shaw et al. (2007) evaluated emulsions as carriers of omega-3 fatty acids in food systems. Characterization of emulsion formulations composed of lecithin or lecithin-chitosan, along with menhaden fish oil and $1-20 \%$ corn syrup, at $6 \%$ humidity was conducted during different storage times. After 5 days of storage, hydroperoxide value increased in samples containing fish oil and corn syrup $(1 \%, 2 \%$, and $10 \%)$ and after 10 days it ranges between 120-170 $\mathrm{m} \mu$ for each $\mathrm{kg}$ of oil. This increase was observed in powders regenerated in water and was observed again after 12 days, when levels were between 140-200 $\mu \mathrm{m}$ for each $\mathrm{kg}$ of oil. The lowest peroxide index was related to powdered emulsion samples with 20 and 5\% corn syrup, which were about 7 and 40 mmol for each $\mathrm{kg}$ of oil, respectively, after 30 days. The propanal value in regenerated samples with $20 \%$ corn syrup was determined after 3 and 6 days and after 12 days it was determined to be less than $25 \mu \mathrm{g}$ for each $\mathrm{kg}$ of oil. Peroxides in these samples ranged between 100-200 mmol for each $\mathrm{kg}$ oil (50). Onuki et al. investigated the special effect of unsaturated fatty acids on bilayer phospholipid membranes with small amounts of cholesterol. The results of their study indicate that omega-3 fatty acids increase separation of regular and irregular phases in membranes and that liposomes made of DPPC are very susceptible to fluidity effect of omega-3 fatty acids. Effects of omega-3 fatty acids on DPPC liposomes are significantly higher than oleic acid and stearic acid, which had no effect on DPPC liposomes. By adding cholesterol to the mix, fluidity effects of omega-3 fatty acids weakened. No evidence was presented to show an increase in lipid raft formation caused by omega-3 fatty acids (48).

In a recent study, Barrow et al. stated that the main problem of fortifying food products with fish oil containing EPA and DHA is their instability and that coacervation could be used commercially to fortify food products and drinks with oils containing omega-3 fatty acids. Reports provided by these authors on the comparison of equivalence for bioavailability of microencapsulated omega-3 fatty acids with complex coacervation. Fish oil soft-gel capsules in human demonstrated that bioavailability of microencapsulated omega-3 fatty acids is equal to the method of complex coacervation by soft-gel fish oil capsules (51). The effect of absorption and interaction of omega-3 fatty acids, including docosahexaenoic acid, docosapentaenoic acid, and linoleic acid, on membrane models of dimyristoylphosphatidylcholine (DMPC) through differential scanning calorimetry was investigated by Sarpietro et al. MLVs resulted from pure DMPC, with different molar values of $0.015-0.18$ of omega-3 fatty acids with two endothermic peaks; a small peak in $15 \mathrm{oC}$ and main peak in $24.8 \mathrm{oC}$. The first peak was transferring from the primary gel stage to the ripple state known as the primary stage, and the second or main peak was transferring from the ripple state to the liquid crystal state. All fatty acids under study resulted in shortening of the primary transfer peak and widening of the calorimetric peaks according to the concentration used. The highest reduction was related to DHA acid, followed by DPA acid and LNA respectively (52). The report from Rasti et al. on the physical and chemical stability of liposomes and nanoliposomes containing fish oil, with $40 \%$ DHA and EPA, prepared by conventional and thermal methods in a 10 -month period after storage at $4 \mathrm{oC}$ revealed that the surface charge and stability of liposomes decreases with increasing size. They observed differences in oxidative stability for PUFA because of the use of inorganic solvents for preparing the liposomes (14). In the following study, the effects of spray and freeze drying was compared with spray-freeze-drying methods in microencapsulation of DHA by Karthilk et al. Efficiency of encapsulation (oil retention) in freeze, spray and spray-freeze-drying methods was reported as $73 \%$, 
$83 \%$, and $71 \%$, respectively. The amount of peroxide found in samples powdered by the spray-freeze method after 36 days of storage in $30 \mathrm{oC}$ was about $8 \mathrm{meq} \mathrm{O} / \mathrm{kg}$ and the results for each of samples prepared using freeze and spray drying were similar for approximately $12 \mathrm{meq} \mathrm{O} / \mathrm{kg}$. Therefore, the amount of peroxide in samples prepared using this method after this period of storage in $4 \mathrm{oC}$ differs slightly and can reach approximately 5 meq $\mathrm{O} 2 / \mathrm{kg}(53)$. The study conducted by Hadian et al. (54) revealed that liposomes can play a key role as solubilizing agents in addressing the need for improved delivery of polyunsaturated FAs. DHA/EPA encapsulated nanoliposomes were found to be promising and capable of effective reversal, which merits further investigation. Using the thin layer hydration method (a schematic flow diagram is shown in Figure 1), they produced DHA and EPA-loaded nanoliposomes. Probe ultrasound treatment of pre-formed liposomes facilitates significant loading of DHA and EPA into the nanoliposomal membrane. Transmission electron microscopy (TEM) image of liposomes showed that the liposomes were spherical in shape and maintain high structural integrity (Figure 2) (54).

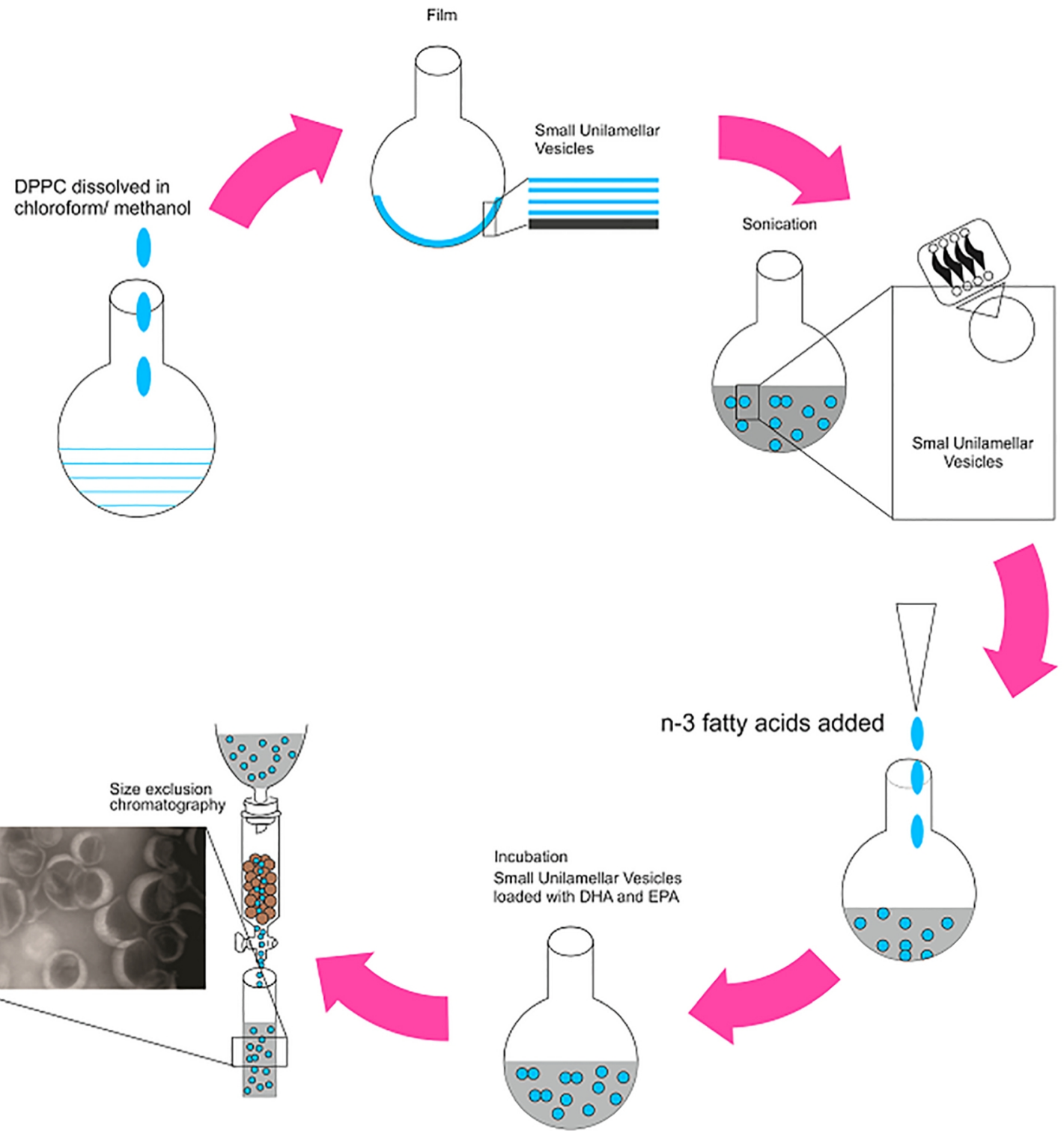

Figure 1. Preparation of DHA and EPA loaded nanoliposomes by the thin film hydration method (54) 


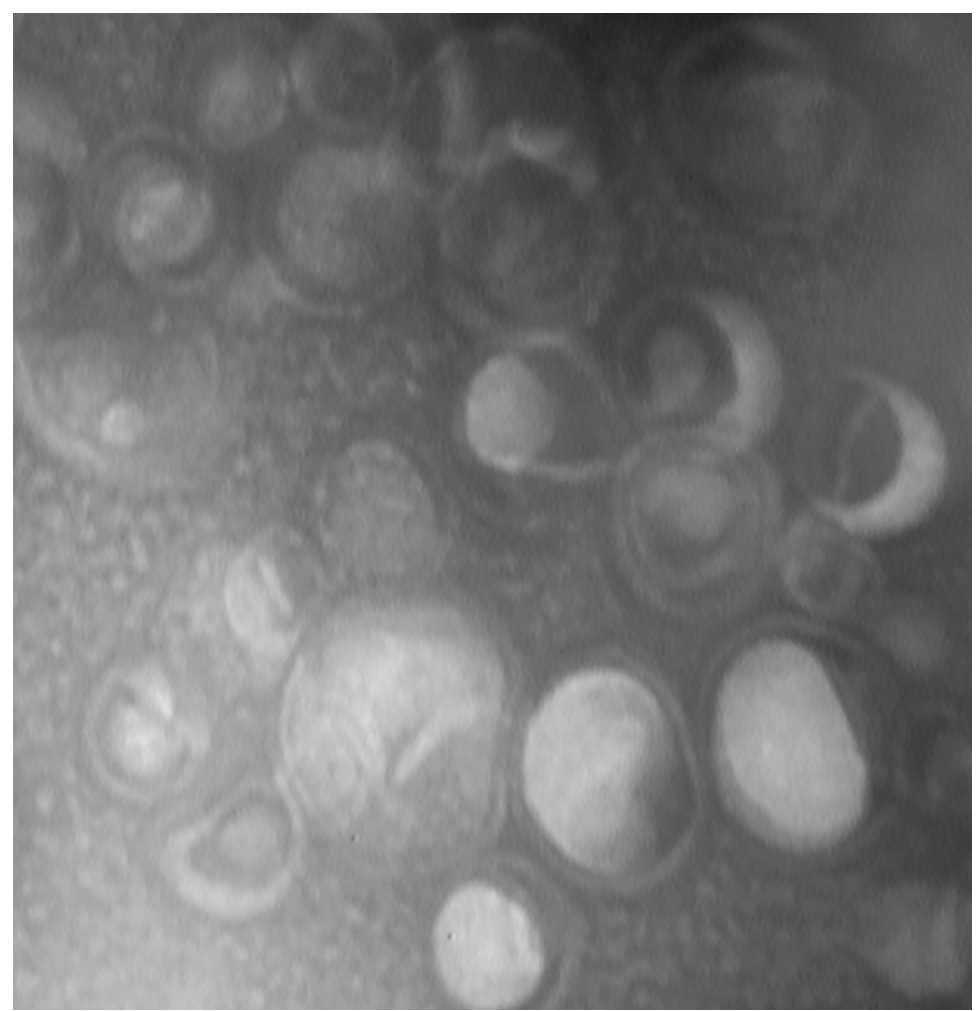

Figure 2. Transmission electron microscopy of DHA and EPA loaded liposomes (Hadian et al. 2014)

\section{Conclusions}

Available evidence suggests that eicosapentaenoic acid and docosahexaenoic acid are useful for preventing various disorders, in particular cardiovascular diseases and inflammatory conditions. The high degree of unsaturation renders the omega 3 fatty acids highly susceptible to oxidation and this oxidation results in a loss of nutritional value and the development of flavors that are unacceptable to consumers. Different strategies, including encapsulation and antioxidants, are used to protect DHA and EPA from oxidation, decreases the odors of volatile oxidation products, and makes them generally palatable. Recent studies have shown that the production of nanoliposomes is considered to be an effective technology for encapsulation of bioactive compounds, as well as enhancing their stability and bioavailability.

\section{Conflict of Interest:}

There is no conflict of interest to be declared.

\section{References}

1) Anderson AS. Fish-risks and benefits. J Hum Nutr Diet. 2004; 17(5): 411-2. doi: 10.1111/j.1365277X.2004.00558.x. PMID: 15357693

2) Swanson D, Block R, Mousa SA. Omega-3 fatty acids EPA and DHA: health benefits throughout life. Adv Nutr. 2012; 3(1): 1-7. doi: 10.3945/an.111.000893, PMID: 22332096.

3) Elizondo E, Moreno E, Cabrera I, Cordoba A, Sala S, Veciana J, et al. Liposomes and other vesicular systems: structural characteristics, methods of preparation, and use in nanomedicine. Prog Mol Biol Transl Sci. 2010; 104: 1-52. doi: 10.1016/B978-0-12-416020-0.00001-2, PMID: 22093216.

4) Nicholson $T$, Khademi $H$, Moghadasian $M H$. The role of marine $n-3$ fatty acids in improving cardiovascular health: a review. Food Funct. 2013; 4(3): 357-65. doi: 10.1039/c2fo30235g, PMID: 23325431.

5) Crepet A, Tressou J, Verger P, Leblanc JC. Management options to reduce exposure to methyl mercury through the consumption of fish and fishery products by the French population. Regul Toxicol Pharmacol. 2005; 42(2): 179-89. doi: 10.1016/j.yrtph.2005.03.006, PMID: 15882918.

6) Von Schacky C. The Omega-3 Index as a risk factor for cardiovascular diseases. Prostaglandins Other Lipid Mediat. 2011; 96(1-4): 94-8. doi: 10.1016/j.prostaglandins.2011.06.008, PMID: 21726658. 
7) Reza Mozafari M, Johnson C, Hatziantoniou S, Demetzos C. Nanoliposomes and their applications in food nanotechnology. J Liposome Res. 2008; 18(4): 309-27. doi: 10.1080/08982100802465941, PMID: 18951288.

8) Siriwardhana N, Kalupahana NS, Moustaid-Moussa N. Health benefits of n-3 polyunsaturated fatty acids: eicosapentaenoic acid and docosahexaenoic acid. Adv Food Nutr Res. 2012; 65: 211-22. doi: 10.1016/B978-0-12-416003-3.00013-5, PMID: 22361189.

9) Zuidam N, Nedovic V. Encapsulation Technologies for Active Food Ingredients and Food Processing, C Springer Science+ Business Media. LLC. 2010.1-400. doi: 10.1007/978-1-4419-1008-0.

10) Brandl M. Liposomes as drug carriers: A technological approach. Biotechnol Annu Rev. 2001; 7: 59-85. doi: 10.1016/S1387-2656(01)07033-8, PMID: 11686049.

11) Khan MA, Shahidi F. Tocopherols and phospholipids enhance the oxidative stability of borage and evening primrose triacylglycerols. J Food Lipids. 2000; 7(3): 143-50. doi: 10.1111/j.1745-4522.2000.tb00167.x.

12) Barenholz Y. Liposome application: problems and prospects. Curr Opin Colloid Interface Sci. 2001; 6(1): 66-77. doi: 10.1016/S1359-0294(00)00090-X.

13) Lasic DD. Liposomes: from physics to applications: Elsevier Science Ltd; 1993.

14) Rasti B, Jinap S, Mozafari M, Yazid A. Comparative study of the oxidative and physical stability of liposomal and nanoliposomal polyunsaturated fatty acids prepared with conventional and Mozafari methods. Food chem. 2012; 135(4): 2761-70. doi: 10.1016/j.foodchem.2012.07.016, PMID: 22980870.

15) Heurtault B, Saulnier P, Pech B, Proust J-E, Benoit J-P. Physico-chemical stability of colloidal lipid particles. Biomaterials. 2003; 24(23): 4283-300. doi: 10.1016/S0142-9612(03)00331-4, PMID: 12853260.

16) Wallace J, McCabe A, Robson P, Keogh M, Murray C, Kelly P, et al. Bioavailability of n-3 polyunsaturated fatty acids (PUFA) in foods enriched with microencapsulated fish oil. Ann Nutr Metab. 2000; 44(4): 157-62, PMID: 11111130.

17) Shahidi F, Finley JW. Omega-3 fatty acids: chemistry, nutrition, and health effects: American Chemical Society; 2001.

18) Baum SJ, Kris-Etherton PM, Willett WC, Lichtenstein AH, Rudel LL, Maki KC, et al. Fatty acids in cardiovascular health and disease: a comprehensive update. J Clin Lipidol. 2012; 6(3): 216-34. doi: 10.1016/j.jacl.2012.04.077, PMID: 22658146.

19) Rogers LK, Valentine CJ, Keim SA. DHA supplementation: current implications in pregnancy and childhood. Pharmacol Res. 2013; 70(1): 13-9. doi: 10.1016/j.phrs.2012.12.003, PMID: 23266567, PMCID: PMC3602397.

20) Kamal-Eldin A. Lipid oxidation pathways: AOCS Press; 2003.

21) Belitz H, Grosch W, Schieberle P, Burghagen M. Food Chemistry, Springer; 1071 p. ISBN: 3540408185. 2004. doi: 10.1007/978-3-540-69934-7.

22) De Lorgeril M, Salen P. New insights into the health effects of dietary saturated and omega-6 and omega-3 polyunsaturated fatty acids. BMC med. 2012; 10(1): 50. doi: 10.1186/1741-7015-10-50, PMID: 22613931, PMCID: PMC3394202.

23) Jafari SM, Assadpoor E, He Y, Bhandari B. Encapsulation efficiency of food flavours and oils during spray drying. Drying Technology. 2008; 26(7): 816-35. doi: 10.1080/07373930802135972.

24) Fathi M, Mozafari M, Mohebbi M. Nanoencapsulation of food ingredients using lipid based delivery systems. Trends Food Sci Tech. 2012; 23(1): 13-27. doi: 10.1016/j.tifs.2011.08.003.

25) Desai KGH, Jin Park H. Recent developments in microencapsulation of food ingredients. Drying technology. 2005; 23(7): 1361-94. doi: 10.1081/DRT-200063478.

26) Arab-Tehrany E, Jacquot M, Gaiani C, Imran M, Desobry S, Linder M. Beneficial effects and oxidative stability of omega-3 long-chain polyunsaturated fatty acids. Trends Food Sci Tech. 2012; 25(1): 24-33. doi: 10.1016/j.tifs.2011.12.002.

27) Patel A, Velikov KP. Colloidal delivery systems in foods: A general comparison with oral drug delivery. LWT-Food Sci Technol. 2011; 44(9): 1958-64. doi: 10.1016/j.lwt.2011.04.005.

28) Olson F, Hunt C, Szoka F, Vail W, Papahadjopoulos D. Preparation of liposomes of defined size distribution by extrusion through polycarbonate membranes. Biochim Biophys Acta. 1979; 557(1): 9-23. 10.1016/0005-2736(79)90085-3, PMID: 95096.

29) Taylor TM, Davidson PM, Bruce BD, Weiss J. Ultrasonic spectroscopy and differential scanning calorimetry of liposomal-encapsulated nisin. J Agric Food Chem. 2005; 53(22): 8722-8. doi: 10.1021/jf050726k, PMID: 16248577.

30) Mozafari MR. Nanocarrier technologies: frontiers of nanotherapy: Springer; 2006. 
31) Perrie Y, Pharmaceutics TR. Drug delivery \& targeting. London: Pharmaceutical Press; 2010.

32) Torchilin V, Weissig V. Liposomes: A practical approach: Oxford University Press; 2003.

33) Meure LA, Foster NR, Dehghani F. Conventional and dense gas techniques for the production of liposomes: a review. AAPS PharmSciTech. 2008; 9(3): 798-809. doi: 10.1208/s12249-008-9097-x, PMID: 18597175, PMCID: PMC2977034.

34) Balon K, Riebesehl B, Müller B. Drug liposome partitioning as a tool for the prediction of human passive intestinal absorption. Pharm Res. 1999; 16(6): 882-8. doi: 10.1023/A: 1018882221008, PMID: 10397609.

35) Hem S, Feldkamp J, White J. Basic chemical principles related to emulsion and suspension dosage forms. The theory and practice of industrial pharmacy, Lachman, L, Lieberman, HA, Kanig, JL, Editors. 1986: $100-22$.

36) Nutan MT, Reddy IK. General principles of suspensions. Pharmaceutical Suspensions: Springer; 2010. p. 39-65.

37) Lichtenberg D, Barenholz Y. Liposomes: preparation, characterization, and preservation. Methods Biochem Anal. 1988; 33: 337-462. PMID: 3282152.

38) Basu SC, Basu M. Liposome methods and protocols: Springer Science \& Business Media; 2002.

39) Schnitzer E, Pinchuk I, Lichtenberg D. Peroxidation of liposomal lipids. Eur Biophys J. 2007; 36(4-5): 499-515. doi: 10.1007/s00249-007-0146-2, PMID: 17380326.

40) Konings AW. Lipid peroxidation in liposomes. Liposome technology. 1984; 1: 139-61.

41) Drusch S, Regier M, Bruhn M. Recent advances in the microencapsulation of oils high in polyunsaturated fatty acids. Novel Technologies in Food Science: Springer; 2012. p. 159-81.

42) Nara E, Miyashita K, Ota T, Nadachi Y. The Oxidative Stabilities of Polyunsaturated Fatty Acids in Salmon Egg Phosphatidylcholine Liposomes. Fisheries science. 1998; 64(2): 282-6.

43) Nacka F, Cansell M, Gouygou J-P, Gerbeaud C, Méléard P, Entressangles B. Physical and chemical stability of marine lipid-based liposomes under acid conditions. Colloids Surf B Biointerfaces. 2001; 20(3): 257-66. doi: 10.1016/S0927-7765(00)00205-8.

44) Nacka F, Cansell M, Méléard P, Combe N. Incorporation of $\alpha$-tocopherol in marine lipid-based liposomes: in vitro and in vivo studies. Lipids. 2001; 36(12): 1313-20. doi: 10.1016/S0927-7765(00)00205-8.

45) Moussaoui N, Cansell M, Denizot A. Marinosomes, marine lipid-based liposomes: physical characterization and potential application in cosmetics. Int J Pharm. 2002; 242(1): 361-5. doi: 10.1016/S0378-5173(02)00217-X, PMID: 12176280.

46) Cansell M, Nacka F, Combe N. Marine lipid-based liposomes increase in vivo FA bioavailability. Lipids. 2003; 38(5): 551-9. doi: 10.1007/s11745-003-1341-0. PMID: 12880112.

47) Lyberg A-M, Fasoli E, Adlercreutz P. Monitoring the oxidation of docosahexaenoic acid in lipids. Lipids. 2005; 40(9): 969-79. doi: 10.1007/s11745-005-1458-1, PMID: 16329470.

48) Onuki Y, Morishita M, Chiba Y, Tokiwa S, Takayama K. Docosahexaenoic acid and eicosapentaenoic acid induce changes in the physical properties of a lipid bilayer model membrane. Chem Pharm Bull. 2006; 54(1): 68-71. doi: 10.1248/cpb.54.68, PMID: 16394552.

49) Namani $T$, Ishikawa $T$, Morigaki $K$, Walde P. Vesicles from docosahexaenoic acid. Colloids Surf $B$ Biointerfaces. 2007; 54(1): 118-23. doi: 10.1016/j.colsurfb.2006.05.022, PMID: 16829059.

50) Shaw LA, McClements DJ, Decker EA. Spray-dried multilayered emulsions as a delivery method for omega-3 fatty acids into food systems. J Agric Food Chem. 2007; 55(8): 3112-9. doi: 10.1021/jf063068s, PMID: 17371041.

51) Barrow CJ, Nolan C, Holub BJ. Bioequivalence of encapsulated and microencapsulated fish-oil supplementation. J Funct Foods. 2009; 1(1): 38-43. doi: 10.1016/j.jff.2008.09.006.

52) Sarpietro MG, Rocco F, Micieli D, Giuffrida MC, Ottimo S, Castelli F. Absorption of omega-3 fatty acids by biomembrane models studied by differential scanning calorimetry. Thermochimica Acta. 2010; 503: 5560. doi: 10.1016/j.tca.2010.03.007.

53) Karthik P, Anandharamakrishnan C. Microencapsulation of docosahexaenoic acid by spray-freeze-drying method and comparison of its stability with spray-drying and freeze-drying methods. Food and Bioprocess Technology. 2013; 6(10): 2780-90. doi: 10.1007/s11947-012-1024-1.

54) Hadian Z, Sahari MA, Moghimi HR, Barzegar M. Formulation, characterization and optimization of liposomes containing eicosapentaenoic and docosahexaenoic acids; A methodology approach. Iran J Pharm Res. 2014; 13(2): 393-404. PMID: 25237335, PMCID: PMC4157015. 